\title{
A Cognitive Modeling of Space using Fingerprints of Places for Mobile Robot Navigation
}

\section{Conference Paper}

\section{Author(s):}

Tapus, Adriana; Siegwart, Roland

Publication date:

2006

Permanent link:

https://doi.org/10.3929/ethz-a-010079366

Rights / license:

In Copyright - Non-Commercial Use Permitted 


\section{A Cognitive Modeling of Space using Fingerprints of Places for Mobile Robot Navigation}

\author{
Adriana Tapus ${ }^{\dagger}$ \\ Ecole Polytechnique Fédérale de Lausanne (EPFL) \\ Autonomous Systems Lab \\ 1015, Lausanne, Switzerland \\ adriana.tapus@ieee.org
}

\author{
Roland Siegwart \\ Ecole Polytechnique Fédérale de Lausanne (EPFL) \\ Autonomous Systems Lab \\ 1015, Lausanne, Switzerland \\ roland.siegwart@epfl.ch
}

\begin{abstract}
In this work we address the problem of perception, spatial cognition and topological navigation for a mobile robot. The objective of this work is to enable the navigation of an autonomous mobile robot (or vehicle) in an indoor (or outdoor) structured environment without relying on maps a priori learned and without using artificial landmarks. A new method for incremental and automatic topological mapping and global localization using fingerprints of places is presented. The fingerprint-based representation permits a reliable, compact and distinctive environment-modeling. Experimental results for mapping indoor and outdoor environments with a mobile robot and a "SMART" vehicle, both equipped with a multi-sensor system composed of two $180^{\circ}$ laser range finders and an omnidirectional camera are also reported.
\end{abstract}

Index Terms - fingerprints of places, topological navigation, cognitive mapping, multi-modal perception

\section{INTRODUCTION}

A robust navigation system requires a spatial model of physical environments as a metric $[1,3,5]$ or topological map $[11,14]$. Approaches using metric maps are suited when it is necessary for the robot to know its location accurately in terms of metric coordinates. However, the state of the robot can also be represented in a more qualitative manner, similar to the way humans do it. The information can be stored as cognitive maps - term introduced for the first time in [15] which permit an encoding of the spatial relations between relevant locations in the environment. This has led to the concept of topological representation. The topological map can be viewed as a graph of places, where at each node the information concerning the visible landmarks and the way to reach other places, connected to it, is stored. The topological representation is compact and allows high-level symbolic reasoning for map building and navigation.

In order to have a robust and reliable framework for navigation (i.e. in order to move within an environment, manipulate objects in it, avoid undesirable collisions, etc.) space cognition, perception, localization and mapping are all needed. The objective of this work is to enable autonomous navigation without relying on maps a priori learned and without using artificial landmarks. Therefore, this paper describes a new method for incremental and automatic topological mapping and global localization with POMDP (Partially Observable Markov Decision Processes) using fingerprints of places. One of the main problems in topological map building is to detect when a new node should be added in the map. Our approach relies on a heuristic that detects whether the current location of the robot is similar to a mapped one or not. The proposed method permits a reliable and distinctive environment model that can be globally handled in an efficient way.

Various methods have been proposed to represent environments in the framework of autonomous navigation, from precise geometric maps based on raw data or lines to purely topological maps using symbolic descriptions. Each of these methods is optimal with respect to some characteristics but can be very disappointing with respect to others. Although literature related to SLAM (Simultaneous Localization and Mapping) is very vast, we only concentrate here on papers that have directly influenced our thinking and research work.

Topological approaches to SLAM attempt to overcome the drawbacks of geometric methods (e.g. problems concerning the global distinctiveness and global consistency) by modeling space using graphs. Significant progress has been made since the seminal paper by Kuipers [8], where, an approach based on concepts derived from a theory on human cognitive mapping is described as the body of knowledge representing large scale space. Kortenkamp and Weymouth in [7] have also used cognitive maps for topological navigation. They defined the concept of gateways which have been used to mark the transition between two adjacent places in the environment. The model described in [4] represents the environment with the help of a Generalized Voronoi Graph (GVG) and localizes the robot via a graph matching process. This approach has been extended to H-SLAM (i.e. Hierarchical SLAM) in [10], by combining the topological and feature-based mapping techniques. In [16], Tomatis et al. have conceived a hybrid representation, similar to the previously mentioned work, comprising of a global topological map with local metric maps associated to each node for precise navigation. Topological maps are less complex and permit more efficient planning than metric maps. Moreover, they are easier to generate. Maintaining global

\footnotetext{
$\dagger$ Adriana Tapus was with Ecole Polytechnique Fédérale de Lausannne, 1015-CH. She is now with the Robotics Research Lab / Interaction Lab, Computer Science Department, University of Southern California, Los Angeles, USA; e-mail: adriana.tapus@iee.org.
} 
consistency is also easier in topological maps compared to metric maps. However, the main problems to deal with, when working with topological maps are the perceptual aliasing (i.e. observations at multiple locations are similar) and the automatic establishment of a minimal topology (nodes).

Our method uses fingerprints of places to create a topological model of the environment. The fingerprint approach, by combining the information from all sensors available to the robot, reduces perceptual aliasing and improves the distinctiveness of places. The main contribution of this paper is the construction of a topological mapping system combined with the localization technique, both relying on fingerprints of places. This fingerprint-based approach yields a consistent and distinctive representation of the environment and is extensible in that it permits spatial cognition beyond just pure navigation.

The rest of the paper is structured as follows. Section II presents a short review of the fingerprint concept. Section III is dedicated to the new topological navigation system with fingerprints of places. Experimental results are presented in Section IV. The systems (indoor and outdoor) use both, two $180^{\circ}$ laser range finders and an omni-directional camera for feature extraction. Finally, Section V draws conclusions and discusses further work.

\section{FINGERPRINTS OF PLACES}

Representing and interpreting a scene from the environment is a hard task. Humans use various sensory cues to extract crucial information from the environment. This is processed in the cortex of the brain in order to obtain a highlevel representation of what has been perceived.

With a view of having robots as companion of humans, we are motivated towards developing a knowledge representation system along the lines of what we know about us. While recent research has shown interesting results, we are still far from having concepts and algorithms that represent and interpret space, coping with the complexity of the environment.

The fingerprint of a place concept is used here. Fingerprints of places (i.e. circular list of significant features around the robot) have been proven to be a very promising approach towards effective place characterization and hence environment modelling $[9,12,13]$. In this work, we choose to use as significant feature: colour bins and vertical edges from the visual information and corners from the laser scanner. Therefore, this multi-modal, feature based representation of space reduces the perceptual aliasing and improve the distinctiveness of space.

\section{TOPOLOGICAL NAVIGATION}

Navigation described by Gallistel in [6], as the capacity to localize itself with respect to a map, is an elementary task that a mobile and autonomous robot must carry out. To navigate reliably in indoor or outdoor environments a mobile robot must know where it is. For this, the robot needs to construct or maintain a spatial representation of the environment. Here, we approach the SLAM (Simultaneous Localization and Mapping) problem that is of a "chicken and egg" nature - to localize the robot, a map is necessary and to update a map the position of the mobile robot is needed.

\section{A. Topological Mapping}

While navigating in the environment, the robot first creates and then updates the global topological map. One of the main issues in topological map building is to detect when a new node should be added in the map. Most of the existing approaches to topological mapping place nodes periodically in either space (displacement, $\Delta d$ ) or time $(\Delta t)$ or alternatively attempt to detect important changes in the environment structure. Any of these methods cannot result in an optimal topology. In contrast, the approach presented in this work is based directly on the differences in the perceived features.

Instead of adding a new node in the map by following some fixed rules (e.g. distance, topology) that limit the approach to indoor or outdoor environments, the method described in this work introduces a new node into the map whenever an important change in the environment occurs. This is possible using the fingerprints of places. A heuristic is applied to compare whether a new location is similar to the last one that has been mapped. Thus, a new node is introduced in the topological map just when important changes into the environment occur. With this, at the end, each node will be composed of a set of similar fingerprints of places. In order to compact even more the current representation, a unique identifier named the mean fingerprint is generated. This technique of clustering fingerprints of places into a single representation enables the construction of a very distinctive and compact representation of the environment. Therefore, a new node contains all posterior knowledge about the environment until the previous node. A more detailed presentation is given in one of our previous works [12]. The incremental nature of the approach permits incremental additions to the map and yields the most up-to-date map at any time.

\section{B. Topological Localization with POMDP}

Finding an efficient solution to the robot localization problem is necessary for the robots to be integrated into our daily lives. Most tasks for which robots are well suited demand a high degree of robustness in their localizing capabilities. A series of localization techniques based on the fingerprint concept have been already presented in [13]. These approaches perform a fingerprint-matching operation so as to localize the robot. The matching methods compare the observed features encoded in the fingerprints of places with the map fingerprints. Only the extereoceptive sensory information contained in fingerprints of places is used for matching, without taking into account the motion of the robot and the previous estimation.

Hence, for topological navigation, a Partially Observable Markov Decision Process (POMDP) model [2] is used here. The POMDPs integrate both the motion and sensor reports data to determine the pose distribution. Thus, by adding the 
motion information to the system, new knowledge about the robot's position is acquired. The probability of being in a place is calculated in function of the last probability distribution, and the current action and observation.

A POMDP is defined as $\langle S, A, T, O\rangle$, where: $S$ is a finite set of environment states; $A$ is a finite set of actions; $T\left(s, a, s^{\prime}\right)$ is a transition function between the environment states based on the action performed; $O$ is a finite set of possible observations; $O S$ is an observation function. With this information, the probability of being in a state $s$ ' (belief state of $s^{\prime}$ ) after having made observation $o$, while performing action $a$, is given by:

$$
S E_{S^{\prime}}^{t+1}=\frac{O S\left(o, s^{\prime}\right) \sum_{S \in S} T\left(s, a, s^{\prime}\right) S E_{S}^{t}}{P\left(o \mid a, S E^{t}\right)}
$$

where $S E_{S}^{t}$ is the belief of state $S$ at the last step, $S E^{t}$ is the belief state vector at the last step, and $P\left(o \mid a, S E^{t}\right)$ is the normalizing factor.

The key idea is to compute a discrete approximation of a probability distribution over all possible poses in the environment. An important feature of this localization technique is the ability to globally localize the robot within the environment. More details about this approach are given in [2].

In our approach, the set of observations $O$ is composed of the fingerprints of places generated by the robot in the environment. These observations are very distinctive since distinctiveness is one of the main characteristics of the fingerprints of places. An observation contains information given by the extereoceptive sensors and designates a subset of the world state. The information for the observation function $O S$ within the topological framework is given by the fingerprint matching algorithm: Global Alignment with Uncertainty [13].

\section{Map Update}

While navigating in the environment, the robot first creates and then updates the global topological map. By using a POMDP (Partially Observable Markov Decision Process), a discrete approximation of a probability distribution over all possible poses in the environment is computed. The entropy of a probability distribution is used here. The lower the value is, the more certain the distribution is. When the robot is "confused", the entropy is high. So the POMDP is confident about its state if the entropy is smaller than a fixed threshold.

Therefore, the strategy of updating the map will be the following:

a) When the entropy of the belief state is low enough, the map will be updated and so the fingerprint and the uncertainty of the features will also be updated.

b) If the entropy is above the threshold, then the updating will not be allowed, and the robot will try to reduce the entropy by continuing the navigation with localization.
When the robot feels confident concerning its state, it can decide if an extracted feature is new by comparing the observed fingerprint to the fingerprint from the map, corresponding to the most confident state. This can happen either in an unexplored portion of the environment, or in a known portion where new features appear due to the environmental dynamics. When a feature is re-observed, the uncertainty of the feature from the map fingerprint is weight averaged with the uncertainty of the extracted one. The weight depends on the type of feature. Since the extraction of features with the laser scanner is more robust than the ones extracted with the camera, a higher weight is given to them. Otherwise, if the robot does not see an expected feature the uncertainty is decreased. When the uncertainty of a feature from a map fingerprint is below a minimum threshold, than the feature is deleted, allowing in this way for dynamics in the environment.

\section{Closing the Loop}

One fundamental problem in SLAM is the identification of a place previously visited, if the robot returned to it. This is known as the closing the loop problem since the robot's trajectory loops back on itself. Thus, for topological maps, this means that if a place (i.e. a node) has been visited before, and the robot returns to it, the robot should detect it (see Figure 1).

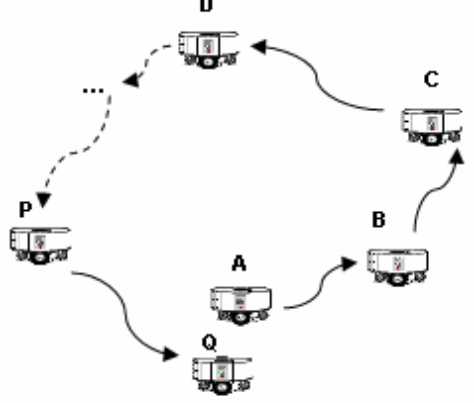

Figure 1: Loop Closing Problem. The robot starts in place A and after moving through the environment arrives in place Q. The question to answer is: Has the robot returned to an already visited place or not? (i.e. Is place A equivalent to place Q?)

Contrary to other methods used for solving this problem, based usually on the perception, loops are identified and closed with the help of the localization techniques. In order to accomplish consistency of the topological map, a method similar to the one described in [16] is used. In this work the method employed is a non-explicit loop closing algorithm. Our loop closing method is based on the localizer (i.e. the POMDP). The robot is moving through the environment and incrementally builds the topological map. As soon as the robot returns in an already visited place (i.e. node) the probability distribution potentially should split up. Two candidates hypotheses should appear: one for the new place (i.e. node) currently created by the robot (e.g. in Figure 1, node $Q$ ) and another one for the previously created node already present in the map (e.g. in Figure 1, node $A$ ). As soon as the POMDP is unconfident, the algorithm tracks the two highest probability distributions showing that the distribution diverged in two peaks. A loop is thus identified if the probability distribution 
given by the localizer converges in two peaks that move in the same direction. In order to detect where the loop was closed, the two hypotheses are backtracked with localization until a single one remains.

\section{EXPERIMENTAL RESULTS}

Our approach for topological SLAM using the fingerprint of places technique has been implemented and evaluated in various real world indoor and outdoor environments. In this section we present some of the indoor experiments carried out with our indoor robot, a fully autonomous mobile robot and the first attempts for outdoor topological mapping using the "SMART" vehicle (Daimler-Chrysler). Both mobile platforms (indoor and outdoor) are equipped with two $180^{\circ}$ laser range finders and an omni-directional camera. The omni-directional camera system uses a mirror-camera system to image $360^{\circ}$ in azimuth and up to $110^{\circ}$ in elevation.

The first set of experiments demonstrates the robustness of the mapping module in two indoor real world scenarios and the first attempts to map urban outdoor environments. In particular, it illustrates the construction of distinctive and compact maps (composed only of local features, which is an advantage of this fingerprint-based mapping technique).

\section{A. Indoor Topological Mapping}

The first indoor experiment was conducted in a portion of our institute building shown in Figure 2 and the second experiment was performed in another building from our campus (see Figure 3(a)). The first test setup was the following: the robot started at the point $\mathrm{S}$ and ended at the point E, as illustrated in the Figure 2, the distance traveled being of $75 \mathrm{~m}$. For the second test the robot traveled a distance of $67 \mathrm{~m}$. While the robot explored the environment, it recorded, at every $\Delta d$ (distance) (e.g. in our case $d=15 \mathrm{~cm}$ ), data readings from sensors (i.e. an image from the omnidirectional camera and a scan from the laser scanner) in order to extract the fingerprints. The robot has a 'mid-line following' behavior in the hallways and 'center of the free space' behavior in the open spaces. We assume that the position in the room with the maximum free space around it, is the one with the highest probability of extracting numerous and characteristic features. This ensures high distinctiveness of the observation. The map building process was performed off-line. The threshold $\theta$, defined as the maximum allowable dissimilarity and used for automatic mapping is calculated experimentally. It is calculated for a small portion of the environment (i.e. $5 \mathrm{~m}$ ), so that the map obtained matches the real structure of the environment. Once this threshold is determined, it is fixed for the rest of the indoor experiments.

Figure 2(b) shows the topological map obtained by the system in the first test environment (i.e. in our laboratory), superimposed on an architectural sketch of the environment. The resulting map is composed of 20 nodes as shown in the Figure 2. Each node is represented by a mean fingerprint which is an aggregation of all the fingerprints composing the respective node. Typically, the nodes are positioned in the rooms and in the hallway.

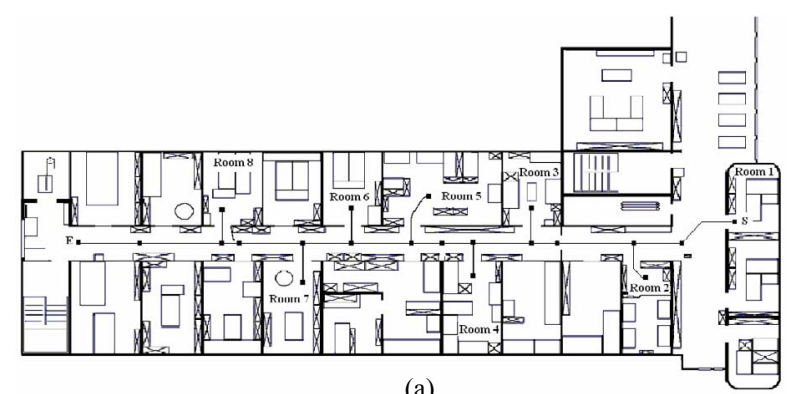

(a)

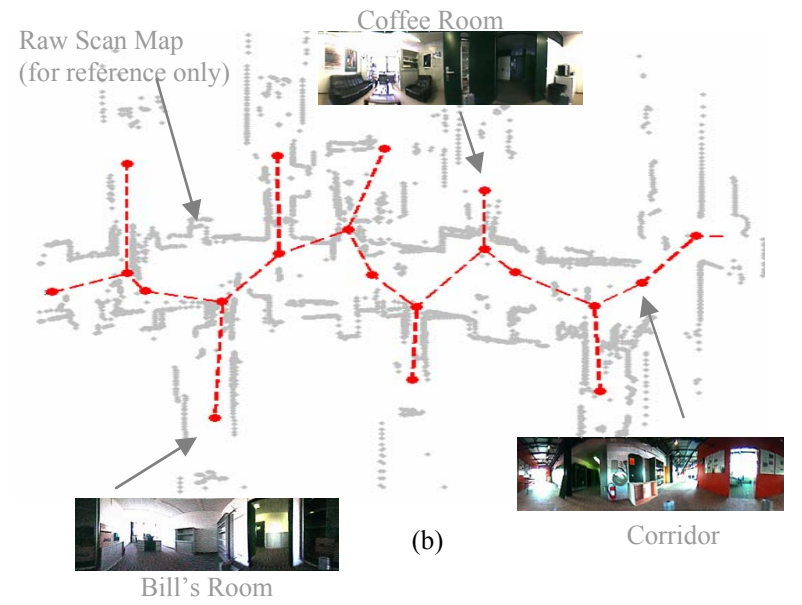

Figure 2: (a) Floor plan of the first environment where the experiments have been conducted. The robot starts at the point $\mathrm{S}$ and ends at the point $\mathrm{E}$. The trajectory length is $75 \mathrm{~m}$. During this experiment, the robot collected 500 data sets (i.e. images and scans) from the environment. The extracted topological map is superimposed on an architectural sketch of the environment. (b) The extracted topological map given by our method, superimposed on the raw scan map.

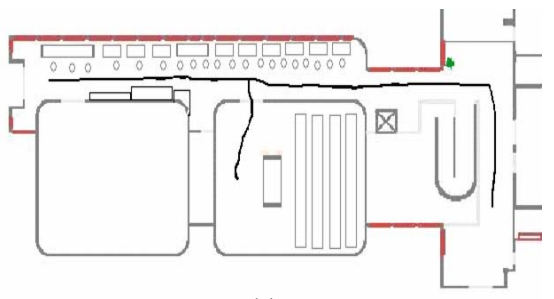

(a)

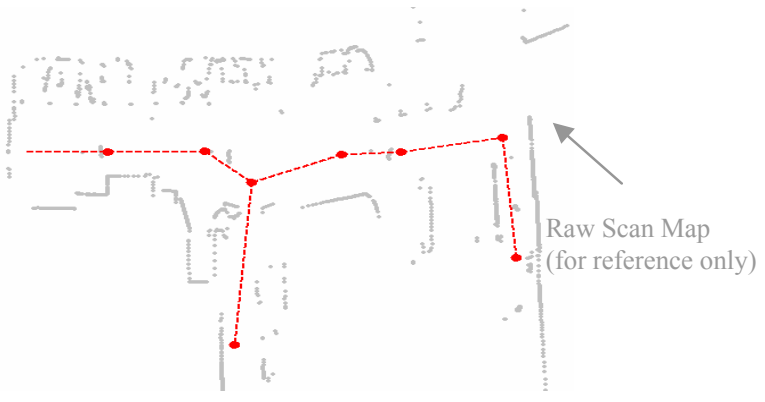

(b)

Figure 3: (a) The second test environment with the trajectory traveled by the robot. (b) The map of the second test environment with the graph representing the topological map. 
The doors of some rooms remained closed at the time of experimentation; this explains why no node is present in front of the respective rooms (see Figure 2).

Figures 3(a) - (b) show the second test environment with the corresponding topological map, formed using the approach outlined in this work. The mapping system added a new node automatically each time a very distinctive measure (i.e. distinctive fingerprint) was encountered. The graph-like map thus obtained contains 8 nodes, as shown in Figure 3(b). The same threshold used for the first test (threshold calculated experimentally) was employed here also, indicating the robustness of the overall method.

The representations thus obtained (see Figure 2 and 3(b)) reproduce correctly the structure of the physical space, in a manner that is compatible with the topology of the environment. They also permit a distinctive modeling of it. It is important to mention that the maps are obtained by using only locally distinctive features composing the fingerprints of places.

\section{B. First Attempts to Outdoor Topological Mapping}

Compared with indoor environments, urban outdoor environments present many challenges for an autonomous vehicle. Coarse localization is often available from GPS. Most of the time, it is more useful to know the position of the robot with respect to buildings, trees, intersections, etc., than the exact latitude and longitude. In order to validate and to show the robustness of our approach, we also tested it in an outdoor environment. The approach has been tested in a part of our campus (highly structured environment), shown in Figure 4, on a $1.65 \mathrm{~km}$ of trajectory. The system mounted on the "SMART" vehicle acquired data, both from the lasers and omni-directional camera every $110 \mathrm{~ms}$. A new threshold for outdoor environments was calculated experimentally in a small portion of the campus. Different thresholds can be used in function of the granularity of the environment that it is desired. High granularity maps, with numerous nodes, may be obtained by setting small thresholds. Alternatively, setting high values for the threshold yields maps with fewer nodes (low granularity). The outdoor threshold for obtaining high granularity maps is the same as the one used for indoor environments. For getting maps with fewer nodes, the outdoor threshold is set three times bigger than the indoor threshold. We have obtained a map composed of 209 nodes for a high granularity and a map of 64 nodes containing only the big changes in the environment (i.e. intersections, new buildings, etc). A small example is depicted in Figure 6(b), which represents a low granularity topological map obtained for a $200 \mathrm{~m}$ section of the environment (i.e. the zoomed view of the magnifying glass shown in Figure 4(a)). The map contains 7 nodes. It can be noticed that the nodes are usually placed in front of buildings, at the crossings and when "big" changes occur (e.g. a building disappears from the field of view of the vehicle and driving signs, lamp-spots and trees appear). The map thus obtained for the entire trajectory shown in Figure 4 is compatible with the structure of the outdoor environment, taking into account the trees, the buildings and the lamp-posts.

\section{Indoor Localization with POMDP}

The quality of the topological maps obtained with our fingerprint-based technique, can be evaluated by testing the localization on it. Localization experiments were conducted so as to show this. To test the localization, more than 1000 new fingerprint samples, acquired while the robot was travelling new paths of $250 \mathrm{~m}$, were used to globally localize the robot

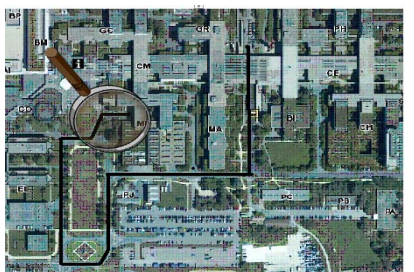

(a)

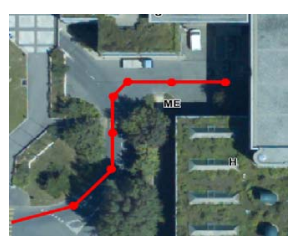

(b)
Figure 4: (a) The outdoor test environment (a part of the EPFL campus) with the trajectory of $1.65 \mathrm{~km}$ long traveled by the Smart vehicle. The magnifying glass represents the part of the environment used for the outdoor topological map exemplification; (b) The low granularity outdoor topological map superimposed on an architectural sketch of a part of the EPFL campus.

with the POMDP. A mission is considered successful if the place found, which corresponds to the world state with the highest probability, is the same with the correct node in the real world.

TABLE 1: Summary of the indoor localization experiments.

\begin{tabular}{|l|l|}
\hline Fingerprints & 1024 samples \\
\hline Distance Travelled & $250 \mathrm{~m}$ \\
\hline Scenarios & $10 / 10$ \\
\hline Kidnapping & $7 / 7$ \\
\hline Fingerprint Matching (GA) & $81 \%$ \\
\hline POMDP localization & $100 \%$ \\
\hline
\end{tabular}

The results are summarized in Table 1. It can be noticed that the results with the POMDP localization have given for the set of scenarios tested in this work a percentage of successful matches of $100 \%$. The kidnapping problem (i.e. recovering from a lost position - the robot thinks that it is in a position where it is not) has also been tested. This was performed seven times and the robot succeeded to recover all the seven times, after one or two steps because of the very distinctive observations that corresponds to the fingerprints.

\section{Closing the Loop}

The localization with POMDP is also used for identification of loops. As explained earlier, the robot moves through the environment and incrementally builds the topological map. The loop closing problem was tested 5 times in different situations within the environment. The robot succeeded to close the loop in all the situations. Figure 5 shows only a simple example that is explained below. In Figure 5, it can be noticed that the robot started in the corridor, in point S. It traveled in the corridor till the door that separates the two hallways was detected (i.e. important change into the environment - node N1), continued in the corridor (i.e. node N2), then entered and went out the Room 3 (i.e. node N3). Once it returned in the corridor, the robot turned left and 
entered in an already visited place, corresponding to node $N 2$. The robot temporarily creates a new node $N 4$. As soon as the robot returned in an already visited place, the POMDP became unconfident and the probability distribution divided in two possible candidate states. Two hypotheses appeared: one for the new place (i.e. node N4 circled in red on Figure 5) currently created by the robot and another one for the previously created node already present in the map (i.e. node N2). The automatic mapper is turned off. The robot moved toward node $N 1$ and labels it at node N5. Node N5 was very similar to node $N 1$, and the correct match is made. A loop is thus identified if the probability distribution given by the localizer converges in two peaks. In order to detect where the loop is closed, the automatic mapping system is turned off and the two hypotheses are backtracked with localization until a single one remains. In the present case, this occurred when node $N 5$ was detected. At that point the robot realized that node N4 is node N2 and that node N5 is node N1. Thus, the loop was closed correctly. In order to make use of the information obtained when a place is revisited, the map is updated. The nodes $N 1$ and N2 are updated with the data brought by the revisited nodes $N 5$ and $N 4$, respectively.

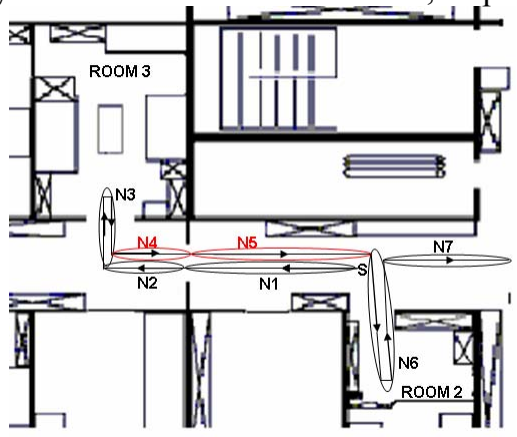

Figure 5: Loop Closing: Shows the directed path that the robot traveled. The robot starts in point $\mathrm{S}$. It can be noticed that the robot arrives in a visited place (i.e. node N4) once it goes out the office (ROOM 3 ) and goes to the left, revisiting again node $\mathrm{N} 4$

As explained earlier, due to the fact that the offices are quite small, the fingerprints of places are very similar, and thus a single node per room is enough. Since a node contains a posterior knowledge about its environment and is the aggregation of all the fingerprints of places between the last node and the current place where an important change into the environment occurred, closing the loop problem does not appear in these cases (i.e. when one node per office is sufficient).

\section{CONCLUSIONS AND FUTURE WORKS}

This paper presented a new technique for topological SLAM using fingerprints of places. A fingerprint of a place provides a compact and distinctive methodology for space representation and place recognition - it permits encoding of a huge amount of place-related information in a single circular sequence of features. This representation is suitable for both indoor and outdoor environments. The experiments verify the efficacy and reliability of our approach. The POMDP localization shown here improves the previously results obtained. Adding the motion of the robot enables to decrease further the pose uncertainty to a level that could never be reached by fingerprint matching alone. A success rate of $100 \%$ was obtained for the tests performed in this work. However, the approach has to be more extensively tested in different types of environment in order to make a real estimation of the quality of the method. In this work, lowlevel features (such as vertical edges) have been used. An interesting extension of the model is the addition of other modalities and features to the fingerprint framework (e.g. auditory, smell, or higher level features such as doors, table, fridge, etc). This will help to improve the reliability and accuracy of the method and to add semantics to it.

\section{ACKNOWLEDGMENTS}

This work was supported by the European project BIBA IST2001-32115 EU project.

\section{REFERENCES}

[1] Arras, K.O. and Siegwart, R, Feature Extraction and Scene Interpretation for Map-Based Navigation and Map Building, In Proceedings of the Symposium on Intelligent Systems and Advanced Manufacturing, Pittsburgh, USA, October 13-17,1997.

[2] Cassandra, A., Kaelbling, L., et al(1996). Acting under Uncertainty: Discrete Bayesian Models for Mobile-Robot Navigation. IEEE International Conference on Robotics and Automation (ICRA), Osaka, Japan.

[3] Castellanos J.A., and Tardos J.D. (1999), Mobile Robot Localization and Map Building: Multisensor Fusion Approach, Kluwer.

[4] Choset, H., and Nagatani, K.(2001), Topological Simultaneous Localization and Mapping (SLAM): Toward Exact Localization Without Explicit Localization, IEEE Trans. On Robotics and Automation, Vol 17, No.2, April.

[5] Dissanayake, Newman, Clark, Durrant-Whyte and Csorba (2001), A Solution to the Simultaneous Localization and Map Building (SLAM) problem, IEEE Trans. On Robotics and Automation, Vol 17, No.3, June.

[6] Gallistel, R. (1990). The Organization of Learning. M. Press. Cambridge, MA

[7] Kortenkamp, D. and Weymouth, T. (1994), Topological mapping for mobile robots using a combination of sonar and vision sensing, In Proceedings of AAAI-94, Seattle, WA.

[8] Kuipers, B. J. (1978), Modeling Spatial Knowledge, Cognitive Science, 2: 129-153, 1978.

[9] Lamon, P., Tapus A., Glauser E., Tomatis N., Siegwart R. (2003), Environmental Modeling with Fingerprint Sequences for Topological Global Localization, Proceedings of the IEEE/RSJ International Conference on Intelligent Robot and Systems(IROS), Las Vegas, USA

[10]Lisien, B., et al. (2003), Hierarchical Simultaneous Localization and Mapping, In Proceedings of the IEEE/RSJ International Conference on Intelligent Robot and Systems, Las Vegas, USA, October 27-30.

[11]Owen, C. and Nehmzow, U.(1998), Landmark-based navigation for a mobile robot, in : Meyer, Berthoz, Floreano, Roitblat and Wilson (Eds.), From Animals to Animate 5, Proceedings of SAB'98, MIT Press, Cambridge, MA, pp. 240-245.

[12]Tapus, A., and Siegwart, R., (2005) Incremental Topological Mapping with Fingerprints of Places, In Proceedings of the IEEE/RSJ International Conference on Intelligent Robot and Systems (IROS), Edmonton, Canada.

[13]Tapus, A., Tomatis, N. and Siegwart, R., (2004) Topological Global Localization and Mapping with Fingerprint and Uncertainty. In Proceedings of the ISER, Singapore, June 2004.

[14]Thrun, S. (1998), Learning metric-topological maps for indoor mobile robot navigation. In Artificial Intelligence 99(1):21-71.

[15]Tolman, E. C. (1948), Cognitive maps in rats and men, Psychological Review, 55:189-208.

[16]Tomatis, N., I. Nourbakhsh, and R. Siegwart (2003). Hybrid simultaneous localization and map building: a natural integration of topological and metric. Robotics and Autonomous Systems, 44:3-14. 